Pacific

Journal of

Mathematics

OBSERVATIONS ON LICKORISH KNOTTING OF CONTRACTIBLE 4-MANIFOLDS

Charles Livingston 


\title{
OBSERVATIONS ON LICKORISH KNOTTING OF CONTRACTIBLE 4-MANIFOLDS
}

\author{
Charles Livingston
}

\begin{abstract}
Lickorish has constructed large families of contractible 4manifolds that have knotted embeddings in the 4-sphere and has also shown that every finitely presented perfect group with balanced presentation occurs as the fundamental group of the complement of a knotted contractible manifold. Here we make a few observations regarding Lickorish's construction, showing how to extend it to construct contractible 4manifolds which have an infinite number of knotted embeddings and also to construct knotted embeddings of the Mazur manifold for which the complement has trivial fundamental group.
\end{abstract}

In his recent paper, Lickorish [Li] describes a clever construction yielding for each finitely presented perfect group $G$ with balanced presentation a compact contractible 4-manifold $M_{G}$ with two embeddings in $S^{4}$, one for which the complement is diffeomorphic to $M_{G}$ and the other with complement having fundamental group $G$. Here we make several observations based on Lickorish's work. With minor modifications, we use the notation of $[\mathbf{L i}]$ throughout.

Observation 1. The construction can be modified to assure that:

(1) For each group $G$ there is an infinite family of $M_{G}$ having the desired pair of embeddings and

(2) For different groups $G$ the constructed infinite families have no elements in common.

Proof. We let $M_{0}$ be the Mazur manifold [Ma] with Kirby diagram as shown in Figure 1. (The curves $\gamma$ and $\gamma^{\prime}$ are extraneous for now.) Since $M_{0}$ embeds in $S^{4}$ with simply connected complement (as in $[\mathbf{L i}], M_{0} \times I \cong B^{5}$ ), the manifold $M_{G}$ of the construction can be replaced with the boundary connected sum $M_{G} \#{ }_{\partial} M_{0}$. This manifold will still have two embedding into $S^{4}$, one with contractible complement and the other with complement having fundamental group $G$. Note that this manifold is not diffeomorphic to $M$ since the boundary has changed by forming the connected sum with the boundary of the Mazur manifold, which Mazur showed is not $S^{3}$. By repeating this process one can easily build the desired families of examples; 
for instance, arrange that for the first group $G$ all the boundaries have a prime number of summands, for the second group $G$ arrange that all have a prime squared number of summands, etcetera.

\section{Figure 1.}

Following Observation 2 we will give examples showing that groups with the desired properties exist.

Observation 2. If the finitely generated perfect group $G$ with balanced presentation maps onto a nontrivial finite quotient of a 2-knot group then there exists an infinite family of embeddings, $\left\{\phi_{i}\right\}$, of the manifold constructed by Lickorish, $M_{G}$, into $S^{4}$ distinguished by $\pi_{1}\left(S^{4}-\phi_{i}\left(M_{G}\right)\right)$.

Proof. Let $K$ be the 2-knot and let $H=\pi_{1}\left(S^{4}-K\right) / N$ be the finite quotient. Let $\rho: G \rightarrow H$ be the given surjective homomorphism. Also, let $x$ be a meridian of $K$ and let $\bar{x}$ denote its image in $H$.

Pick an element $g \in G$ such that $\rho(g)=\bar{x}$. It can be arranged in the construction of $M_{G}$ that $g$ is among the generators of $G$ in the balanced presentation: Simply add a generator $z$ to the balanced presentation along with the relation $z=g$, with $g$ written in terms of the generators of the initial balanced presentation.

It now follows from the initial construction of $M_{G}$ as the complement of $X_{G}$ that the meridian of the 2-handle of $M_{G}$ corresponding to the generator $z$ represents $g \in G=\pi_{1}\left(X_{G}\right)$. To construct a new embedding of $M_{G}$ into $S^{4}$, tie the knot $K$ as a local knot in the given 2-handle of $M_{G}$. This does not change $M_{G}$ but changes the fundamental group of $X_{G}$; the new group is constructed from the free product $G * \pi_{1}\left(S^{4}-K\right)$ by identifying $g \in G$ with $x \in \pi_{1}\left(S^{4}-K\right)$. We denote this group by $G_{1}$ and also write it as $G *_{Z} \pi_{1}\left(S^{4}-K\right)$ though it is not an amalgamated product in the case that $g$ has finite order in $G$. (It is not clear that this new group is not isomorphic to $G$.) 
There are two homomorphisms of $\pi_{1}\left(S^{4}-K\right)$ to $H$ : The first is the projection, $p$, the second factors through the cyclic group $Z$, mapping the meridian to $\bar{x}$. Denote this second map by $q$. The maps $\rho * p$ and $\rho * q$ each determine homomorphisms of $G_{1}$ to $H$. These homomorphisms are distinct as one is surjective when restricted to the image of $\pi_{1}\left(S^{4}-K\right)$ and the other is not surjective when restricted to this subgroup. (Note that $H$ is perfect since it is a quotient of $G$ and hence is not cyclic.)

We first observe that these two embeddings cannot be isotopic; if they were there would be an isomorphism from $G$ to $G *_{Z} \pi_{1}\left(S^{4}-K\right)$ carrying the meridian representing to $g$ to the meridian $m^{\prime}$ representing $g=x$. Thus there would be a group isomorphism from $G$ to $G *_{Z} \pi_{1}\left(S^{4}-K\right)$ sending $g$ to $g=x$. However that cannot be as, by the above argument, $G$ and $G *_{Z} \pi_{1}\left(S^{4}-K\right)$ have different numbers of homomorphisms onto $H$ sending these preferred meridians to $\bar{x}$. (Notice that since $H$ is finite there is a finite number of such homomorphisms.)

By repeating the construction of locally knotting the 2-handle of $M$ using $K$, a sequence of nonisotopic embeddings of $M$ into $S^{4}$ is constructed.

We cannot show that the sequence of fundamental groups of the complements are all distinct, but by counting homomorphisms to $H$ it follows that some subsequence must be distinct; the number of homomorphisms onto $H$ goes to infinity since after adding $n$ knots to the band there are at least $2^{n}$ homomorphisms onto $H$.

Examples. This simplest example of Observation 2 occurs with the binary icosahedral group, $H(2,3,5)$, the perfect group with 120 elements representing the fundamental group of +1 surgery on the trefoil knot. This group clearly has a balanced presentation and is also a quotient of the trefoil group, which is isomorphic to the fundamental group of the 0-twist spin of the trefoil. More generally, consider the group of the $r$-fold cyclic branched cover of the $(p, q)$-torus knot, denoted $H(p, q, r)$. If $p, q$, and $r$, are pairwise relatively prime, then $H(p, q, r)$ is perfect. Furthermore, according to Gordon, [Go], the $r$-twist spin of the $(p, q)$-torus knot has fundamental group $H(p, q, r) \times Z$, and hence maps onto $H(p, q, r)$. It remains to show that $H(p, q, r)$ has a finite quotient. A presentation of $H(p, q, r)$ is given by $\left\langle x, y \mid x^{p}=y^{q}=(x y)^{r}\right\rangle$. For such groups a nontrivial representation to an alternating group can be constructed. (This was done by Fox in [Fo]; a more accessible reference is Milnor [Mi].)

Observation 3. There exist contractible manifolds built with a single 1handle that possess two embeddings in $S^{4}$, one with simply connected complement and one with nontrivial complementary fundamental group.

Proof. This fact follows from the result of Neuzil $[\mathbf{N e}]$ showing that the Dunce Cap embeds in $S^{4}$ with nonsimply connected complement. The following approach gives us more control over the contractible manifold as well. 
Suppose that $L=L_{1} \cup L_{2}$ is a 2-component link in $S^{3}$ with $L_{1}$ unknotted bounding a trivial slice disk $D_{1}$ in $B^{4}$ and $L_{2}$ slice, bounding a slice disk $D_{2}$ in $B^{4}$. Assume the linking number is 1 . Let $S^{3}$ separate $S^{4}$ into two components, $B_{1}$ and $B_{2}$ and view $D_{1} \subset B_{1}$ and $D_{2} \subset B_{2}$.

As in Lickorish's construction, we let $M=\left(B_{1}-N\left(D_{1}\right)\right) \cup N\left(D_{2}\right)$. This is clearly a contractible 4-manifold that doubles to give $S^{4}$. However, its complement is $X=\left(B_{2}-N\left(D_{2}\right)\right) \cup N\left(D_{1}\right)$. Its group is given by the group of the complement of the slice disk with an added relations coming from adding the 2-handle, $N\left(D_{1}\right)$.

Examples. For any knot $K$, the knot $L_{2}=K \#-K$ is slice with fundamental group of the complement of the slice disk being $\pi_{1}\left(S^{3}-K\right)$. Any element of this group can be represented by an unknot $L_{1}$ in the complement of $L_{2}$. Hence, the groups that arise in this construction include all perfect groups constructed by adding a single relation to a classical knot group. For instance: The fundamental group of a homology 3-sphere built by surgery on a classical knot is the fundamental group of the complement of the embedding of a contractible 4-manifold with one 1-handle into $S^{4}$.

In the previous construction it is not clear that we are constructing distinct embeddings if $L_{2}$ is unknotted; this case is perhaps the most perplexing. We have the following example:

Observation 4. The Mazur Manifold illustrated in Figure 1 has two nonisotopic embeddings into $S^{4}$.

Proof. As described for instance in $[\mathbf{A k}]$, the boundary of the Mazur manifold $M$ has an involution $F$ carrying $\gamma$ to $\gamma^{\prime}$. A handlebody picture of the manifold $M \cup_{i d} M$ is formed from Figure 1 by adding a 2-handle with 0 framing to $\gamma^{\prime}$. Similarly, a handlebody picture of the manifold $M \cup_{F} M$ is formed from Figure 1 by adding a 2-handle with 0 framing to $\gamma$. (In each case a 3- and 4-handle must be added as well.) It is an easy exercise in Kirby calculus $[\mathbf{A K}]$ to see that both are $S^{4}$. Hence, we have two embeddings of $M$ into $S^{4}$.

Clearly, in the first case the curve $\gamma^{\prime}$ is slice in the complement - the 2-handle is added to $\gamma^{\prime}$. In the second case $\gamma^{\prime}$ is not slice in the complement - this is a result of Akbulut, [Ak].

Questions. In the above construction, if $L_{2}$ is unknotted is there an embedding of the constructed Mazur-like manifold into $S^{4}$ with nonsimply connected complement? If the crossing that is not part of the clasp of the attaching map of the 2-handle in Figure 1 is changed the previous argument does not apply - Akbulut has shown that in this case $\gamma$ will be slice. Does this manifold knot in $S^{4}$ ? Does there exist a contractible 4-manifold that does not knot in $S^{4}$ ? 
Acknowledgements. Thanks go to Ray Lickorish, first for identifying this interesting topic, secondly for pointing out some of the issues resolved here, and finally for his reflections on my first attempts at extending his results.

\section{References}

[Ak] S. Akbulut, A fake compact contractible 4-manifold, J. Differential Geom., 33(2) (1991), 335-356, MR 92b:57025, Zbl 0839.57015.

[AK] S. Akbulut and R. Kirby, Mazur manifolds, Michigan Math. J., 26(3) (1979), 259284, MR 80h:57004, Zbl 0443.57011.

[Fo] R. Fox, On Fenchel's conjecture about F-groups, Mat. Tidsskrift, B (1952), 61-65, MR 14,843c, Zbl 0049.15404.

[Fr] B. Freed, Embedding contractible 2-complexes in $E^{4}$, Proc. Amer. Math. Soc., 54 (1976), 423-430, MR 52 \#11915, Zbl 0316.57004.

[Go] C. McA. Gordon, Twist-spun torus knots, Proc. Amer. Math. Soc., 32 (1972), 319322, MR 44 \#5948, Zbl 0231.55006.

[Li] W.B.R. Lickorish, Knotted contractible 4-manifolds in the 4-sphere, Pacific J. Math., 208(2) (2003), 283-290.

[Ma] B. Mazur, A note on some contractible 4-manifolds, Ann. of Math. (2), 73 (1961), 221-228, MR 23 \#A2873, Zbl 0127.13604.

[Mi] J. Milnor, Knots, groups, and 3-manifolds (Papers dedicated to the memory of R.H. Fox), 175-225, Ann. of Math. Studies, 84, Princeton Univ. Press, Princeton, NJ, 1975 .

[Ne] J. Neuzil, Embedding the dunce hat in $S^{4}$, Topology, 12 (1973), 411-415, MR 50 \#14764, Zbl 0271.57004.

Received November 24, 2001 and revised January 6, 2002.

Department of Mathematics

INDIANA UNIVERSITY

BLOOMINGTON, IN 47405

E-mail address: livingst@indiana.edu 